\title{
Effective slip lengths for longitudinal shear flow over partial-slip circular bubble mattresses
}

\author{
Darren Crowdy $\ddagger$ \\ Department of Mathematics, Imperial College London, 180 Queen's Gate, London, \\ SW7 2AZ, United Kingdom \\ E-mail: d.crowdy@imperial.ac.uk
}

\begin{abstract}
The problem of longitudinal shear flow over a circular bubble mattress with partial slip and protrusion angle $90^{\circ}$ is solved in a quasi-analytical fashion by a novel transform scheme recently devised by the author. The general approach can be readily adapted to other mixed boundary value problems. From the analysis explicit approximations for the effective slip lengths are found as a function of the Navier-slip parameter and the area fraction of the surface covered by protrusions. These new approximation formulas for the slip lengths both unify and extend those based on empirical polynomial fits to numerical data given recently by $\mathrm{Ng} \&$ Wang [Fluid Dyn. Res., 43, 065504, (2011)].
\end{abstract}

Keywords: superhydrophobic surface, slip length, bubble mattress, longitudinal flow 


\section{Introduction}

Driven by applications in microfluidics there has been much recent interest in understanding the friction properties of superhydrophobic surfaces $[1,20,14,12,23]$. Typically, these are no-slip surfaces endowed with additional microstructural features such as grooves or holes which, in the so-called Cassie state [19], are occupied by pockets of gas rather than being liquid-filled (as in the Wenzel state). The gas-liquid interfaces, or "bubble" surfaces, spanning the edges of the no-slip surface usually allow some degree of slip. As a result, superhydrophobic surfaces have been observed to reduce fluid drag in microchannels $[16,17,10]$. The drag reduction has strong dependence on the geometry and local slip properties of the gas-liquid interfaces of which the surface is made up [22]. It has therefore become a matter of great practical importance to quantify these slip properties.

The focus of this paper is a class of superhydrophobic surfaces possessing a periodic array of unidirectional grooves or bubbles; see Figure 1 where the case of longitudinal shear flow (i.e., flow along the grooves) is indicated. Suppose the cross-section of some superhydrophobic surface has mean height along the plane $y=0$. For general linear shear flows with shear rate $\dot{\gamma}$ over such a surface the velocity field far from the plane of the surface takes the form

$$
\mathbf{u}=\dot{\gamma}(y+\lambda) \hat{\mathbf{x}}
$$

where $\hat{\mathbf{x}}$ is the flow direction. The constant $\lambda$ is known as the effective slip length and is a measure of the frictional properties of the surface: it is the fictional distance below the surface at which the shear flow would extrapolate to zero. It is an important quantifier of the slip properties of the surface [11]. The slip lengths for longitudinal flow and tranverse flow (i.e., flow across the grooves) are generally very different.

In this paper we are concerned with a theoretical study of the problem of linear shear flow over an array of unidirectional grooves each of width 2 and periodic in the $x$ direction with period $2 l$ for $l>1$. The grooves are spanned by liquid-gas interfaces that are circular arcs with protrusion angle $\theta$ into the liquid and there are no-slip surfaces between these free surfaces. Such a surface is sometimes called a unidirectional bubble mattress [6, 3]. Numerical calculations of the slip lengths associated with such bubble mattresses have been performed by Teo \& Khoo [25] (who used a finite element method) and Ng \& Wang [15] (who used a semi-analytical method based on a collocation scheme). The latter authors found the effective slip lengths, for both transverse and longitudinal flow, for arbitrary $\theta$, for arbitrary area fractions $\delta=1 / l$, and allowing for partial slip on the liquid-gas interface. This means that for longitudinal flow the boundary condition on the liquid-gas interface is

$$
w=\mu \frac{\partial w}{\partial n},
$$

where $w(x, y)$ is the axial velocity, $\partial / \partial n$ denotes a surface normal derivative and $\mu$ is the so-called Navier slip parameter. When $\mu=0$ the interface is a no-slip surface; $\mu=\infty$ 


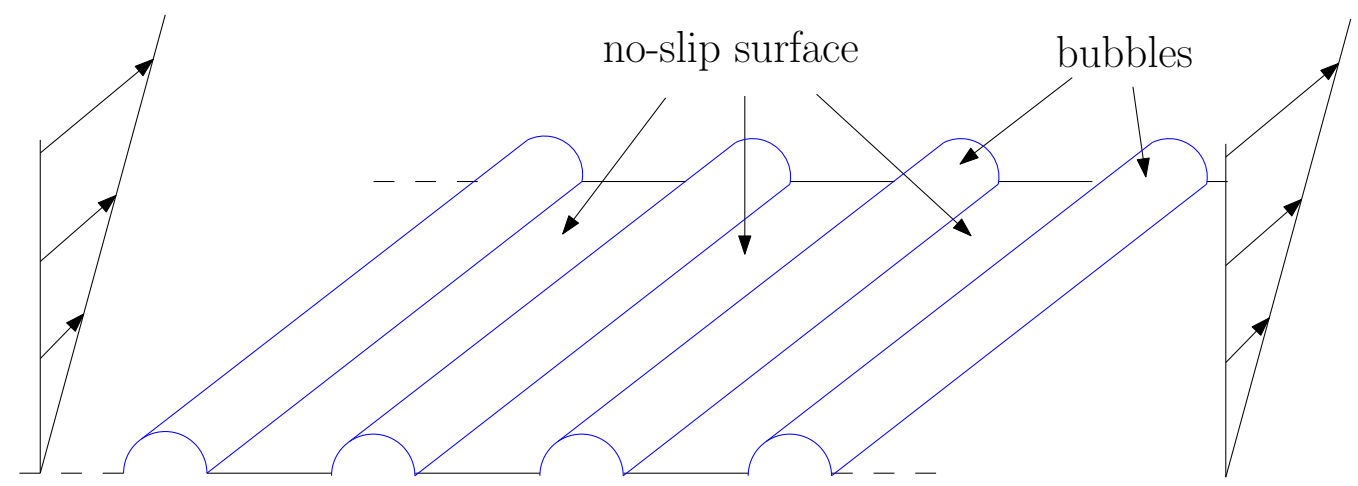

Figure 1. Longitudinal flow along a unidirectional bubble mattress.

corresponds to a no-shear surface and the boundary condition (2) becomes

$$
\frac{\partial w}{\partial n}=0
$$

For the latter case of no-shear conditions on the bubble surfaces a complete analytical characterization of the effective slip properties of such surfaces is known in the dilute limit. Written with the same normalizations used by Ng \& Wang [15], Davis \& Lauga [6] derived the explicit formula

$$
\lambda^{\perp}=M(\theta)=\pi \delta^{2} \int_{0}^{\infty} A(s, \theta) d s,
$$

for the transverse slip-length with no-shear on the bubbles where

$$
A(s, \theta)=\frac{s}{\sinh 2 s(\pi-\theta)+s \sin 2 \theta} \times\left[\cos 2 \theta+\frac{s \sin 2 \theta \cosh s \pi+\sinh s(\pi-2 \theta)}{\sinh s \pi}\right],
$$

where $\delta$ is assumed to be small. For longitudinal shear flow with boundary condition (3) on the bubbles, again in the dilute limit, the author [3] has derived the explicit slip length formula

$$
\lambda^{\|}=N(\theta)=\frac{\pi \delta^{2}}{12}\left[\frac{3 \pi^{2}-4 \pi \theta+2 \theta^{2}}{(\pi-\theta)^{2}}\right],
$$

where $\delta$ is again assumed to be small. To obtain (6) the boundary condition on the liquid-gas interface is taken to be (3). Armed with (5) and (6) the slip length of any linear shear over the surface can be determined as a linear combination of the transverse and longitudinal results. Ng \& Wang [15] made use of the analytical results (4) and (6) to check their numerical calculations in the dilute limit. Sbragaglia \& Prosperetti [21] have carried out a perturbation analysis to find the slip properties for longitudinal flow over close-to-flat bubble profiles (i.e., $\theta \approx 0$ ) in channels. Other theoretical work where slip lengths have been calculated in various settings appears in $[18,13,2,21]$.

It is important to be clear about the distinction between the Navier slip-parameter, denoted here by $\mu$, and the effective slip-length, denoted here by $\lambda$. The parameter $\mu$ in (2) is sometimes called the intrinsic or microscopic slip [15] and is associated with local physico-chemical properties of the surface (e.g., hydrophobicity of the surface 
due to some chemical treatment). The effective slip length, on the other hand, is a more global macroscopic quantity characterizing the gross slip behaviour of a fluid over a heterogeneous surface with patterning, grooves, free spanning surfaces and other assorted local features. Finding the effective slip length as a function of the local slip properties is generally a challenging task since it depends on the nature and geometry of the surface features. Returning to circular, unidirectional bubble mattresses, in the important special case $\theta=90^{\circ}, \mathrm{Ng} \&$ Wang [15] give empirical polynomial fits (to their numerical data) for the effective longitudinal slip lengths in the two limiting cases $\mu=\infty$ and $\mu=0$ :

$$
\begin{aligned}
& \lambda_{\mathrm{Ng}+\text { Wang }}^{\infty}=-0.40 \delta+8.93 \delta^{2}-46.74 \delta^{3}+134.28 \delta^{4}-173.88 \delta^{5}+86.56 \delta^{6}, \\
& \lambda_{\mathrm{Ng}+\text { Wang }}^{0}=-0.005 \delta-1.72 \delta^{2}+0.85 \delta^{3},
\end{aligned}
$$

where superscripts denote the relevant $\mu$-value.

The purpose of the present paper is to describe a new, quasi-analytical approach to solving for the longitudinal slip length when $\theta=90^{\circ}$ for arbitrary $\delta$ and $\mu$. Thus, for the case $\theta=90^{\circ}$, we extend the result (6) beyond the dilute limit and to the case of partial slip on the interface (i.e., arbitrary $\mu$ ). Our analysis gives results for all area fractions $\delta$ in the form of the solution of a simple linear system of small size thereby obviating the need for finite element or boundary element methods. The method here is also very different to the collocation method used by $\mathrm{Ng} \&$ Wang [15]. Our approach is general and can be applied to many other flow scenarios.

As a useful side-product we find new explicit approximation formulas for the longitudinal slip lengths:

$$
\lambda_{\text {approx }}^{\mu}=\frac{3 \alpha \delta(\mu-1)}{\alpha^{2}(1-\mu)+3(1+\mu)}, \quad \alpha=\frac{\pi \delta}{2} .
$$

This compact formula gives excellent agreement to the actual slip length values for $\delta$ approximately in the range $\delta \in[0,0.9]$. Notice that (9) is a function of both the local Navier slip parameter $\mu$ and the area fraction $\delta$; the formula therefore both unifies and extrapolates the empirical formulas (8) to arbitrary $0 \leq \mu<\infty$.

From a mathematical perspective our results have additional interest in showcasing a new, flexible constructive solution method for problems of this type: the bubble mattress problem is solved by means of a novel transform technique recently derived by the author $[4,5]$. The approach is very general and offers a way to solve boundary value problems for harmonic fields in multiply connected circular domains, i.e., domains whose boundaries are circular arcs and straight lines. The transform method formulated in $[4,5]$ is an extension (to circular, multiply connected domains) of a transform approach to harmonic boundary value problems in convex polygons first presented by Fokas \& Kapaev [8]. All these transform techniques fit into a much broader collective known as the unified transform method pioneered by A. S. Fokas [7] (often called the Fokas method) and which applies not only to linear boundary value problems but also to initial value problems for nonlinear integrable partial differential equations. 
As a matter of fact, the bubble mattress problem, in the special case $\mu=\infty$, is included in [5] to illustrate the scope of the general method described there. The present paper shows how to generalize that example study to incorporate boundary condition (2) for arbitrary $\mu \geq 0$ corresponding to differing degrees of partial slip on the interface.

\section{Mathematical formulation}

Let the axial (longitudinal) velocity be $w(x, y)$ so that in Cartesian coordinates

$$
\mathbf{u}=(0,0, w(x, y)) \text {. }
$$

It is convenient to rotate by $90^{\circ}$ the view given in Figure 1 and to assume that the flow takes place in the right half channel region $x>0,-l<y<l$ (with $l>1$ ) exterior to the unit disc $|z|<1$ as shown in Figure 2 where the periodic array of bubbles now sits along the $y$-axis. In the absence of a pressure gradient, $w(x, y)$ is harmonic in the flow region and it is convenient to introduce the complex potential

$$
h(z)=\chi+\mathrm{i} w,
$$

where $\chi$ is the harmonic conjugate to $w$. The boundary conditions on the edges of the period window are that

$$
\chi=\left\{\begin{array}{rr}
-c, & y=-l, \\
c, & y=l,
\end{array}\right.
$$

with no-slip on the solid surface,

$$
w=0, \quad x=0, \quad 1<|y|<l,
$$

and a Navier slip condition on the circular bubble interface,

$$
w=\mu \frac{\partial w}{\partial n}, \quad|z|=1,
$$

where $\mu>0$ is the Navier slip parameter.

The boundary condition (13) allows us, by the Schwarz reflection principle, to analytically continue the function $w(z)$ into the full channel region $-l<y<l$ exterior to the unit disc $|z|<1$. Let this doubly connected circular region be denoted by $D$ as shown in Figure 3.

We decompose $h(z)$ in the form

$$
h(z)=\mathrm{i} z+\mathrm{i} \lambda \operatorname{coth}\left[\frac{\pi z}{2 l}\right]+H(z),
$$

where $H(z)$ and the slip length $\lambda$ are to be determined. Provided that the function $H(z)$ is analytic and single-valued in $D$, and decaying as $|z| \rightarrow \infty$, then (15) is consistent with the required far-field shear $(28)$ or, in this case,

$$
w \rightarrow x+\lambda, \quad \text { as } x \rightarrow \infty,
$$

where we have set the far-field shear rate to be unity. 


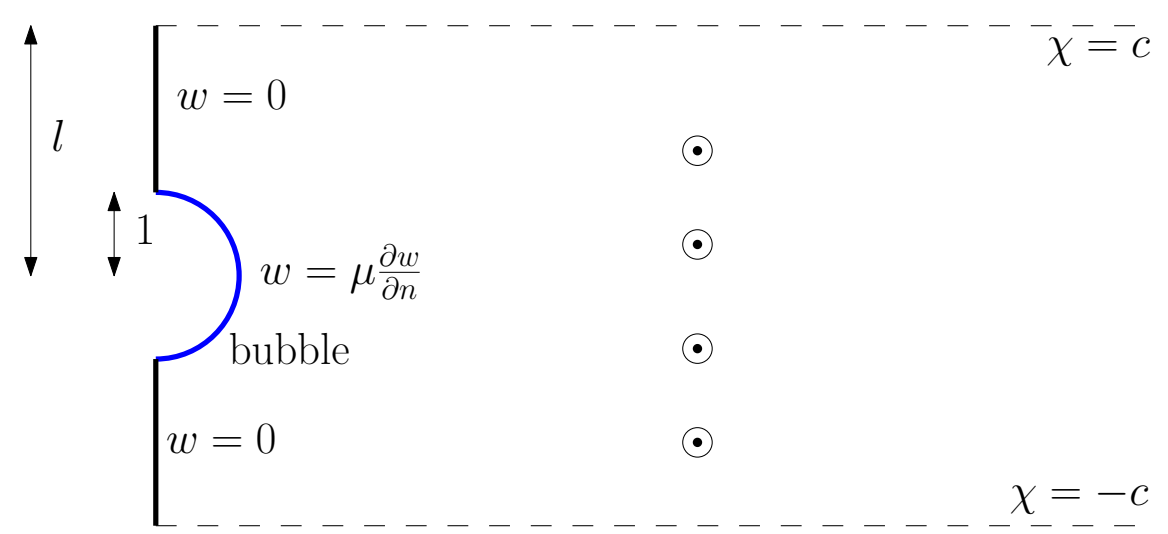

Figure 2. Typical period window of half-period $l$ for longitudinal shear flow past a unit radius bubble protruding with angle $\theta=90^{\circ}$ and exhibiting partial slip with Navier slip coefficient $\mu$. The area fraction $\delta=1 / l$ is the fraction of the period cell occupied by the protruding bubble.

We can deduce some more properties of $H(z)$. Since, by the flow symmetry, on $\bar{z}=z$, we have $\chi=0$ then

$$
\operatorname{Re}[h(z)]=0=\operatorname{Re}[H(z)]
$$

and we deduce that

$$
\bar{H}(z)=-H(z) .
$$

This relation holds off the line $\bar{z}=z$ by analytic continuation. Furthermore, from (13), on $\bar{z}=-z$ outside the bubble we also have $w=0$ so that

$$
0=H(z)-\overline{H(z)}=H(z)-\bar{H}(-z)=H(z)+H(-z),
$$

where we have used (18), which implies that $H(z)$ is odd, i.e.,

$$
H(-z)=-H(z) \text {. }
$$

Again, this relation holds off the line $\bar{z}=-z$ by analytic continuation.

It is convenient to rewrite the Navier slip condition (14) in terms of the complex potential $w(z)$. Now

$$
\frac{\partial w}{\partial n}=\nabla w \cdot \mathbf{n}=\operatorname{Re}\left[\left(\frac{\partial w}{\partial x}-\mathrm{i} \frac{\partial w}{\partial y}\right) z\right],
$$

where $z$ is the complex unit normal to the unit circle. But

$$
\frac{\partial w}{\partial x}-\mathrm{i} \frac{\partial w}{\partial y}=2 \frac{\partial w}{\partial z}=2 \frac{\partial}{\partial z}\left[\frac{h(z)-\overline{h(z)}}{2 \mathrm{i}}\right]=-\mathrm{i} h^{\prime}(z),
$$

so the complex form of the normal derivative is

$$
\frac{\partial w}{\partial n} \mapsto \operatorname{Re}\left[-\mathrm{i} z h^{\prime}(z)\right]=\operatorname{Im}\left[z h^{\prime}(z)\right] .
$$




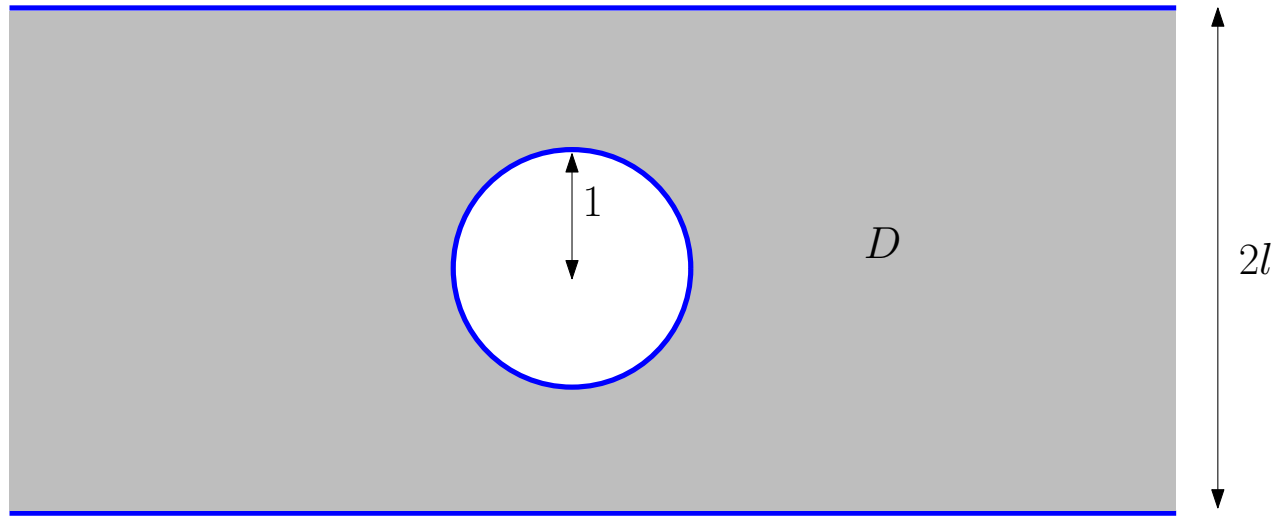

Figure 3. The extended channel region $D$ where $H(z)$ is single-valued and analytic. The transform analysis is carried out for $z \in D$.

The boundary condition on the circular interface $|z|=1$ is then

$$
\operatorname{Im}\left[h(z)-\mu z h^{\prime}(z)\right]=0 .
$$

This implies, on substitution of (15), that on $|z|=1$,

$$
\operatorname{Im}\left[H(z)-\mu z H^{\prime}(z)\right]=\operatorname{Im}[R(z)]+\lambda \operatorname{Im}[S(z)],
$$

where

$$
R(z) \equiv-\mathrm{i}(1-\mu) z, \quad S(z) \equiv-\left[\mathrm{i} \operatorname{coth}\left[\frac{\pi z}{2 l}\right]+\frac{\mathrm{i} \mu \pi z}{2 l} \operatorname{cosech}^{2}\left[\frac{\pi z}{2 l}\right]\right] .
$$

On $y=-l$, where $\bar{z}=z+2 \mathrm{il}$, the boundary condition (12) implies

$$
\mathrm{i} z+\mathrm{i} \lambda \operatorname{coth}\left[\frac{\pi z}{2 l}\right]+H(z)-\mathrm{i}(z+2 \mathrm{i} l)-\mathrm{i} \lambda \operatorname{coth}\left[\frac{\pi(z+2 \mathrm{i} l)}{2 l}\right]+\bar{H}(z+2 \mathrm{i} l)=-2 c
$$

which, by the periodicity of the coth function and use of (18), yields

$$
H(z)-H(z+2 \mathrm{i} l)=-2(c+l) .
$$

On $y=l$, where $\bar{z}=z-2 \mathrm{il}$, the boundary condition (12) implies

$$
\mathrm{i} z+\mathrm{i} \lambda \operatorname{coth}\left[\frac{\pi z}{2 l}\right]+H(z)-\mathrm{i}(z-2 \mathrm{i} l)-\mathrm{i} \lambda \operatorname{coth}\left[\frac{\pi(z-2 \mathrm{i} l)}{2 l}\right]+\bar{H}(z-2 \mathrm{i} l)=2 c
$$

which, on letting $z \mapsto z+2 \mathrm{il}$, produces the same result (28). Since we require $H(z)$ to decay as $|z| \rightarrow \infty(28)$ implies that $c=-l$. Hence $H(z)$ is 2il periodic:

$$
H(z)=H(z+2 \mathrm{i} l) \text {. }
$$

\section{Integral transform representation}

We now summarize the results of [5] where transform pairs are derived that are specially tailored to multiply connected circular geometries like the disc-in-channel geometry $D$ arising here. This new transform method will be used to solve the boundary value 


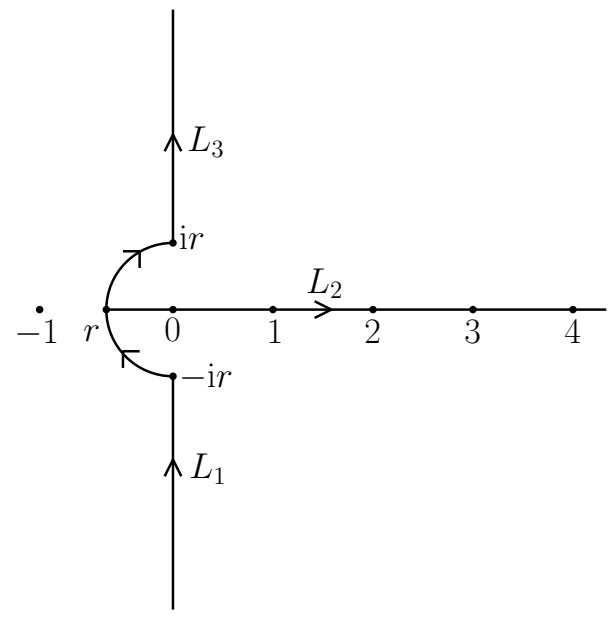

Figure 4. The contours $L_{1}, L_{2}$ and $L_{3}$ for circular edges. We must pick $0<r<1$.

problem for $H(z)$ stated in $\S 2$. The following calculation is a generalization of a similar one featured in [5].

It is established in [5] that we can write the following integral transform representation of the required $H(z)$ for $z \in D$ :

$$
\begin{gathered}
H(z)=\frac{1}{2 \pi} \int_{0}^{\infty} \rho_{11}(k) e^{\mathrm{i} k z} d k+\frac{1}{2 \pi} \int_{0}^{-\infty} \rho_{33}(k) e^{\mathrm{i} k z} d k \\
\quad-\frac{1}{2 \pi \mathrm{i}}\left\{\int_{L_{1}} \frac{\rho_{22}(k)}{1-e^{2 \pi \mathrm{i} k}} \frac{1}{z^{k+1}} d k+\int_{L_{2}} \rho_{22}(k) \frac{1}{z^{k+1}} d k+\int_{L_{3}} \frac{\rho_{22}(k) e^{2 \pi \mathrm{i} k}}{1-e^{2 \pi \mathrm{i} k}} \frac{1}{z^{k+1}} d k\right\},
\end{gathered}
$$

where $\left\{L_{j} \mid j=1,2,3\right\}$ are the contours shown in Figure 4. The three so-called spectral functions are defined to be

$$
\rho_{11}(k)=\int_{-\infty-\mathrm{i} l}^{+\infty-\mathrm{i} l} H(z) e^{-\mathrm{i} k z} d z, \quad \rho_{22}(k)=-\oint_{|z|=1} H(z) z^{k} d z,
$$

and

$$
\rho_{33}(k)=\int_{\infty+\mathrm{i} l}^{-\infty+\mathrm{i} l} H(z) e^{-\mathrm{i} k z} d z
$$

There are other elements of the so-called spectral matrix which are given by

$$
\rho_{21}(k)=\int_{-\infty-\mathrm{i} l}^{+\infty-\mathrm{i} l} H(z) z^{k} d z, \quad \rho_{23}(k)=\int_{\infty+\mathrm{i} l}^{-\infty+\mathrm{i} l} H(z) z^{k} d z,
$$

and

$$
\rho_{12}(k)=\rho_{32}(k)=-\oint_{|z|=1} H(z) e^{-\mathrm{i} k z} d z,
$$

and $\rho_{31}(k)=\rho_{11}(k)$ and $\rho_{13}(k)=\rho_{33}(k)$.

All these spectral functions have special analytical structure, and this can be exploited to determine those spectral functions. This analytical structure is encoded 
in the so-called global relations which can be stated as follows:

$$
\begin{array}{ll}
\rho_{11}(k)+\rho_{12}(k)+\rho_{13}(k)=0, & k \in \mathbb{R}, \\
\rho_{31}(k)+\rho_{32}(k)+\rho_{33}(k)=0, & k \in \mathbb{R},
\end{array}
$$

and

$$
\rho_{21}(k)+\rho_{22}(k)+\rho_{23}(k)=0, \quad k \in-\mathbb{N} .
$$

The global relations (37) and (38) can be analyzed to find the unknown spectral functions. Once found, they can be substituted into (31) to give an integral representation of the sought-after $H(z)$.

\section{Solving for the spectral functions}

We now show how to analyze the global relations to determine the spectral functions. Each of the two global relations (36) and (37) is equivalent to the statement

$$
\int_{-\infty}^{\infty} \tilde{H}(x) e^{-\mathrm{i} k x}\left[e^{-k l}-e^{k l}\right] d x-\oint_{|z|=1} e^{-\mathrm{i} k z} H(z) d z=0, \quad k \in \mathbb{R},
$$

where we have defined

$$
\tilde{H}(x) \equiv H(x-\mathrm{i} l)
$$

and made use of the periodicity property (30). The global relation (38) is equivalent to

$$
\int_{-\infty}^{\infty} \tilde{H}(x)\left[\frac{1}{(x-\mathrm{i} l)^{k}}-\frac{1}{(x+\mathrm{i} l)^{k}}\right] d x-\oint_{|z|=1} H(z) \frac{d z}{z^{k}}=0, \quad k \in \mathbb{N} .
$$

On $y=-l$ we know that

$$
\operatorname{Re}[h(z)]=l .
$$

On substitution of (15) we find that, on this boundary,

$$
\operatorname{Re}[H(z)]=0 .
$$

Therefore, on $y= \pm l$, by the periodicity, we will write

$$
\tilde{H}(x)=\mathrm{i} \Psi(x)
$$

for some real function $\Psi(x)$ to be determined. On the other hand, on $|z|=1$, we write

$$
H(z)=\mathrm{i} \sum_{n \geq 1}\left\{a_{n} z^{2 n-1}+\frac{b_{n}}{z^{2 n-1}}\right\}
$$

for some real coefficients $\left\{a_{n}, b_{n} \in \mathbb{R}\right\}$ to be found. The ansatz (45) satisfies (18) and (20).

(39) can be written as

$$
-2 \sinh (k l) \mathcal{H}(k)=B(k),
$$

where

$$
\mathcal{H}(k) \equiv \int_{-\infty}^{\infty} \mathrm{i} \Psi(x) e^{-\mathrm{i} k x} d x
$$


is recognized as the standard Fourier transform of $\mathrm{i} \Psi(x)$ and

$$
B(k) \equiv \oint_{|z|=1} H(z) e^{-\mathrm{i} k z} d z
$$

It follows that

$$
\mathcal{H}(k)=-\frac{B(k)}{2 \sinh (k l)} .
$$

From an inverse Fourier transform we find

$$
\mathrm{i} \Psi(x)=-\frac{1}{2 \pi} \int_{-\infty}^{\infty} \frac{B(k)}{2 \sinh (k l)} e^{\mathrm{i} k x} d k .
$$

It is clear that we must insist that

$$
B(0)=0
$$

in order to remove the singularity at $k=0$ of the integrand of (50).

Now (41) implies that, for $n \geq 1$,

$$
\oint_{|z|=1} \frac{H(z)}{z^{n}} d z=\int_{-\infty}^{\infty} \mathrm{i} \Psi(x)\left[\frac{1}{(x-\mathrm{i} l)^{n}}-\frac{1}{(x+\mathrm{i} l)^{n}}\right] d x .
$$

But it is easy to show, on use of (45), that

$$
\oint_{|z|=1} \frac{H(z)}{z^{2 n}} d z=-2 \pi a_{n}
$$

Hence (52) implies

$$
a_{n}=-\frac{1}{2 \pi} \int_{-\infty}^{\infty} \mathrm{i} \Psi(x)\left[\frac{1}{(x-\mathrm{i} l)^{2 n}}-\frac{1}{(x+\mathrm{i} l)^{2 n}}\right] d x .
$$

On substitution of (50) into (54) we find

$$
a_{n}=\int_{-\infty}^{\infty} I(k)\left[\frac{B(k)}{2 \sinh (k l)}\right] d k
$$

where

$$
I(k) \equiv \frac{1}{4 \pi^{2}} \int_{-\infty}^{\infty} e^{\mathrm{i} k x}\left[\frac{1}{(x-\mathrm{i} l)^{2 n}}-\frac{1}{(x+\mathrm{i} l)^{2 n}}\right] d x .
$$

An exercise in residue calculus reveals that

$$
I(k)=\left\{\begin{array}{cl}
\frac{\mathrm{i} e^{-k l}(\mathrm{i} k)^{2 n-1}}{2 \pi(2 n-1) !}, & k>0, \\
\frac{\mathrm{i} e^{k l}(\mathrm{i} k)^{2 n-1}}{2 \pi(2 n-1) !}, & k<0 .
\end{array}\right.
$$

On substitution of (57) into (55), and after a change of integration variable so that all integrals are over the positive real $k$-axis, (55) becomes

$$
a_{n}=\frac{\mathrm{i}}{2 \pi} \int_{0}^{\infty} \frac{e^{-k l}(\mathrm{i} k)^{2 n-1}}{(2 n-1) !}\left[\frac{B(k)+B(-k)}{2 \sinh (k l)}\right] d k, \quad n \geq 1 .
$$


But

$$
\begin{aligned}
B(k) & =\oint_{|z|=1} e^{-\mathrm{i} k z} H(z) d z=\oint_{|z|=1} \mathrm{i} e^{-\mathrm{i} k z} \sum_{m \geq 1}\left[a_{m} z^{2 m-1}+\frac{b_{m}}{z^{2 m-1}}\right] d z \\
& =-2 \pi \sum_{m \geq 1} \frac{b_{m}(\mathrm{i} k)^{2 m-2}}{(2 m-2) !}
\end{aligned}
$$

so that

$$
B(k)+B(-k)=-4 \pi \sum_{m \geq 1} \frac{b_{m}(\mathrm{i} k)^{2 m-2}}{(2 m-2) !} .
$$

The system of equations (58) is therefore equivalent to

$$
a_{n}=\sum_{m \geq 1} A_{n m} b_{m}, \quad n \geq 1
$$

where

$$
A_{n m}=-\mathrm{i} \int_{0}^{\infty} \frac{e^{-k l}}{\sinh (k l)} \frac{(\mathrm{i} k)^{2(m+n)-3}}{(2 m-2) !(2 n-1) !} d k .
$$

We have not yet imposed the Navier slip condition on $|z|=1$. It implies additional relations between the coefficients $\left\{a_{n}, b_{n} \mid n \geq 1\right\}$. Suppose that, on $|z|=1, R(z)$ and $S(z)$ have the Laurent expansions

$$
R(z)=\sum_{n \geq 1} r_{n} z^{2 n-1}+\frac{r_{-n}}{z^{2 n-1}}, \quad S(z)=\sum_{n \geq 1} s_{n} z^{2 n-1}+\frac{s_{-n}}{z^{2 n-1}} .
$$

These coefficients can be found analytically from (26) or, more conveniently, they can be readily computed by a Fast Fourier transform. On substitution into (25) and on equating coefficients we find, for $n \geq 1$,

$$
\mathrm{i} a_{n}(1-\mu(2 n-1))+\mathrm{i} b_{n}(1+\mu(2 n-1))=r_{n}-\overline{r_{-n}}+\lambda\left(s_{n}-\overline{s_{-n}}\right) .
$$

This implies

$$
b_{n}=-\left[\frac{1-\mu(2 n-1)}{1+\mu(2 n-1)}\right] a_{n}+p_{n}+\lambda q_{n}, \quad n \geq 1,
$$

where

$$
p_{n}=\frac{r_{n}-\overline{r_{-n}}}{\mathrm{i}(1+\mu(2 n-1))}, \quad q_{n}=\frac{s_{n}-\overline{s_{-n}}}{\mathrm{i}(1+\mu(2 n-1))} .
$$

On use of (66) to eliminate $b_{n}$ from (62) we find

$$
a_{n}=\sum_{m \geq 1} A_{n m}\left[-\left[\frac{1-\mu(2 m-1)}{1+\mu(2 m-1)}\right] a_{m}+p_{m}+\lambda q_{m}\right],
$$

for $n \geq 1$. The additional condition (51) implies that

$$
b_{1}=0
$$

and, hence, that

$$
0=-\left[\frac{1-\mu}{1+\mu}\right] a_{1}+p_{1}+\lambda q_{1} .
$$




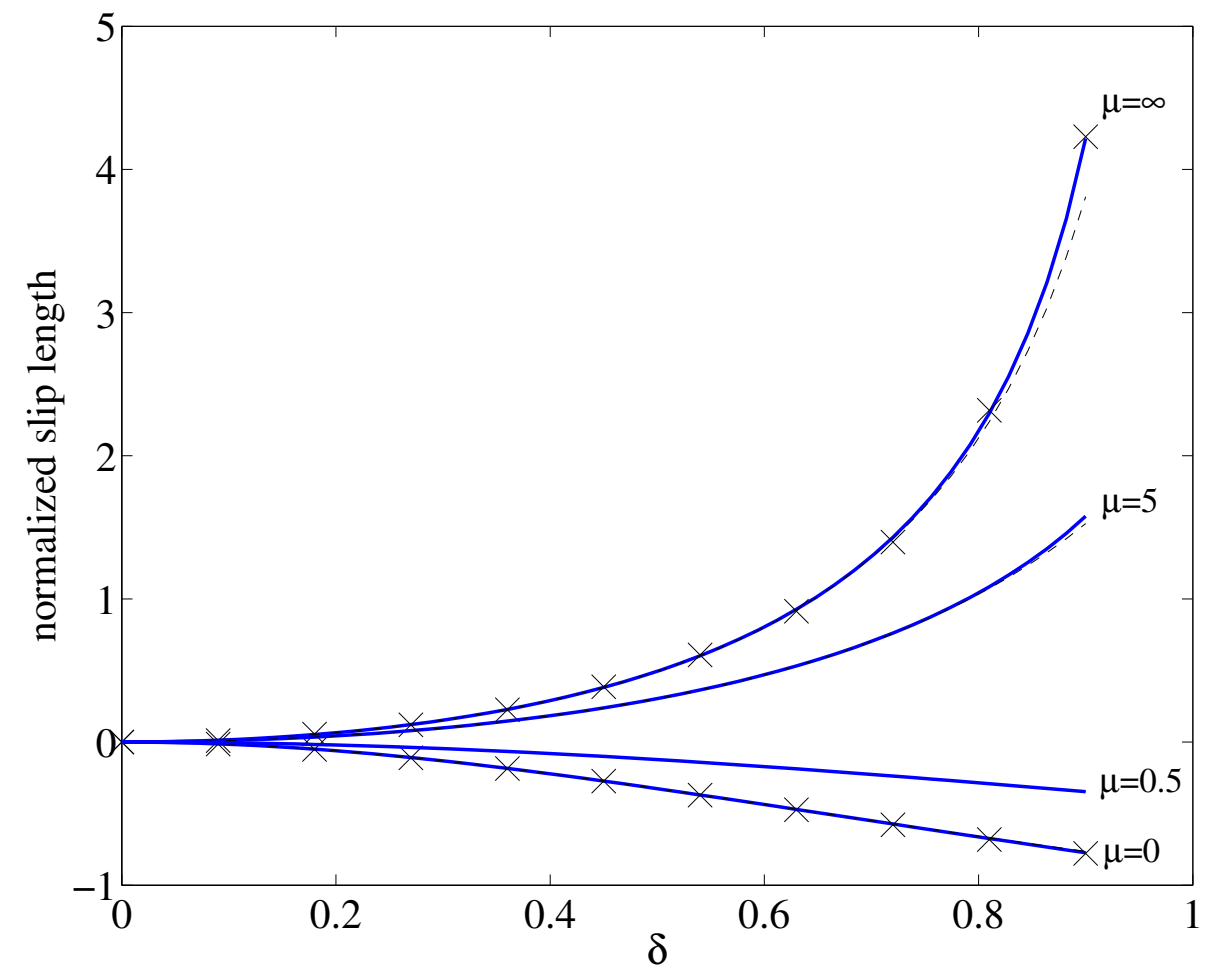

Figure 5. Comparison of slip lengths, for $\mu=0,0.5,5$ and $\infty$ and $0<\delta<0.9$ as given by the transform method (solid lines) and by the approximations (9) (dashed lines). The dashed lines are only detectable to the eye for $\mu=\infty$ and $\delta \in(0.8,0.9)$ reflecting the overall efficacy of the approximations (9). Crosses denote data points given by the empirical fits (8) of [15] for $\mu=0, \infty$.

In summary, the final linear system to be solved for the set of coefficients $\left\{a_{n} \mid n \geq 1\right\}$ and $\lambda$ is

$$
\begin{aligned}
& {\left[\frac{1-\mu}{1+\mu}\right] a_{1}-\lambda q_{1}=p_{1},} \\
& a_{n}+\sum_{m \geq 1} C_{n m} a_{m}-\lambda \sum_{m \geq 1} A_{n m} q_{m}=\sum_{m \geq 1} A_{n m} p_{m}, \quad n \geq 1
\end{aligned}
$$

with

$$
C_{n m}=A_{n m}\left[\frac{1-\mu(2 m-1)}{1+\mu(2 m-1)}\right] .
$$

Once $\left\{a_{n} \mid n \geq 1\right\}$ and $\lambda$ are found, $\left\{b_{n} \mid n \geq 1\right\}$ follow from (66).

\section{Approximate slip length formulas}

Numerical solutions of the linear system (71)-(72) reveal that, for area fractions $\delta$ in the range $[0,0.9]$, the value of $a_{1}$ is small compared to the other terms in (71). With this 
evidence, if we assume that $a_{1}=0$ in (71) we find the following useful approximation formula for $\lambda$ :

$$
\lambda_{\text {approx }}^{\mu}=-\frac{p_{1}}{q_{1}}=-\frac{\oint_{|z|=1}(R(z)-\bar{R}(1 / z)) d z}{\oint_{|z|=1}(S(z)-\bar{S}(1 / z)) d z} .
$$

All contour integrals in this expression can be computed explicitly by the residue theorem to give the result (9). For the cases $\mu=0$ and $\mu=\infty$ we find

$$
\begin{array}{cc}
\lambda_{\text {approx }}^{0}=-\frac{3 \alpha \delta}{3+\alpha^{2}}, & \alpha=\frac{\pi \delta}{2}, \\
\lambda_{\text {approx }}^{\infty}=\frac{3 \alpha \delta}{3-\alpha^{2}}, & \alpha=\frac{\pi \delta}{2},
\end{array}
$$

which are convenient alternatives to the empirical polynomial fits given in (8). For $\mu=\infty$ and $\delta \ll 1$ it can be checked that (75) retrieves the dilute result (6) when $\theta=90^{\circ}$. Figure 5 shows how the slip lengths given by formulas (75) (shown as solid lines) compare to those given by the formulae (8) of $\mathrm{Ng} \&$ Wang [15] (shown as dashed lines). The two approximations are almost indistinguishable for $\delta$ in the range $[0,0.9]$ with minor differences only emerging as $\mu \rightarrow \infty$, and only at sufficiently large $\delta$.

Figure 5 displays data for the two intermediate cases of partial slip with $\mu=0.5$ and $\mu=5$. Results of the full solution from the transform method together with the slip length given by the approximation formula (9) are shown. Once again the approximation is almost indistinguishable from the unapproximated value for area fractions $\delta$ in the range $[0,0.9]$. Finally, the solid curves in Figure 5 giving the values from the full unapproximated solution of the system (71)-(72) agree very well with the empirical fits (8) to numerical data from [15] for $\mu=0, \infty$ across the full range $\delta \in[0,0.9]$ lending us confidence in the efficacy of our approach.

\section{Discussion}

The evidence of the previous section shows that the approximation formula (9) is a concise and useful one for a broad range of area fractions (i.e., not just in the dilute limit) and for all values of the Navier slip parameter $0 \leq \mu<\infty$.

On the other hand, if a calculation of the full unapproximated slip length values is needed or preferred, the system (71)-(72) can be solved very easily using just a handful of lines of code in MATLAB and with almost negligible computational effort. Indeed, even for $\delta \rightarrow 1^{-}$(i.e., the limit of closely packed bubbles) it was found that truncating the linear system (71)-(72) with just 8 modes (so that the size of the linear system is just 9-by-9) gives excellent accuracy (although for $\delta$ very close to unity the numerical approximation eventually deteriorates as must be expected).

Our focus here has been on determining the effective slip lengths, but if values of the axial velocity at points in the flow are needed, for example, these can be readily 
computed once the linear system (71)-(72) has been solved. With $\left\{a_{n} \mid n \geq 1\right\}$ and $\lambda$ determined, the coefficients $\left\{b_{n} \mid n \geq 1\right\}$ follow from (66). This means that $H(z)$ is known on $|z|=1$ from (45) so $\rho_{22}(k)$ can be found from (32), while $\rho_{12}(k)$ and $\rho_{32}(k)$ can be computed from (35) with (37)-(38) then used to determine $\rho_{11}(k)$ and $\rho_{33}(k)$. Thus, all three spectral functions $\rho_{11}(k), \rho_{22}(k)$ and $\rho_{33}(k)$ appearing in the explicit integral representation (31) for $H(z)$ are known. Hence $h(z)$ is computable.

Finite element methods [25], collocation methods based on eigenfunction expansions [15] as well as lattice Boltzmann methods [10,9] have all been deployed for the study of the slip properties of superhydrophobic surfaces. The new transform approach of this paper is distinct from these prior methods and, while not fully analytical since it involves the solution of a small linear system (72), the method requires minimal computational effort to find the effective slip lengths. Finally, the general method used in this paper can be applied to mixed boundary value problems in a broad range of other circular domain geometries [5].

Acknowledgments: The author acknowledges the support of an Established Career Fellowship from the Engineering and Physical Sciences Research Council in the UK and a Wolfson Research Merit Award from the Royal Society.

Data accessibility statement: This article contains no external data.

Competing interest statement: We have no competing interests.

Author's contributions: The single author was solely responsible to all aspects of the article including devising the original concept, developing the analysis, performing all calculations and reportage of the results in this manuscript. He is solely responsible for the writing and preparation of the article.

Funding statement: The research in this article is supported by an Established Career Fellowship from the Engineering and Physical Sciences Research Council (EP/K019430/1).

[1] C. Cottin-Bizonne, J. L. Barrat, L. Bocquet \& E. Charlaix, Low-friction flows of liquid at nanopatterned interfaces, Nat. Mater., 2, 237-240, (2003).

[2] D. G. Crowdy, Frictional slip lengths for a class of unidirectional superhydrophobic surfaces, Phys. Fluids, 23, 072001 (2011).

[3] D.G. Crowdy, Slip length for longitudinal shear flow over a dilute periodic mattress of protruding bubbles, Phys. Fluids, 22, 121703, (2011).

[4] D. G. Crowdy, Fourier-Mellin transforms for circular domains, Comput. Methods. Funct. Theory, (2015); doi: 10.1007/s40315-015-0139-6.

[5] D. G. Crowdy, A transform method for Laplace's equation in multiply connected circular domains, IMA J. Appl. Math., (2015); doi: 10.1093/imamat/hxv019.

[6] A.M.J. Davis \& E. Lauga, Geometric transition in friction for flow over a bubble mattress, Phys. Fluids, 21, 011701, (2009).

[7] A. S. Fokas, A unified approach to boundary value problems, CBMS-NSF Regional Conference Series in Applied Mathematics, No 78, SIAM, Philadelphia, (2008).

[8] A. S. Fokas \& A. A. Kapaev, On a transform method for the Laplace equation in a polygon, IMA J. Appl. Math., 68, 355-408, (2003). 
[9] J. Harting, C. Kumert \& J. Hyväluoma, Lattice Boltzmann simulations in microfluidics: probing the no-slip boundary conditions in hydrophobic, rough and surface nanobubble laden microchannels, Microfluid. Nanofluid., 8, 1-10, (2010).

[10] J. Hyväluoma \& J. Harting, Slip flow over structured surfaces with entrapped microbubbles, Phys. Rev. Lett., 100, 246001, (2008).

[11] K. Kamrin, M.Z. Bazant \& H.A. Stone, Effective slip boundary conditions for arbitrary periodic surfaces: the surface mobility tensor, J. Fluid. Mech., 658, 409-437, (2010).

[12] E. Lauga, M. P. Brenner \& H. A. Stone, Microfluidics: The no-slip boundary condition, Handbook of Experimental Fluid Dynamics, Eds: A. Y. C. Tropea and J. F. Foss, Springer, New York, (2007).

[13] E. Lauga \& H.A. Stone, Effective slip in pressure-driven Stokes flow, J. Fluid Mech., 489, 55-77, (2003).

[14] C. Neto, D. R. Evans, E. Bonaccurso, H.-J. Butt \& V. S. J. Craig, Boundary slip in Newtonian liquids: a review of experimental studies, Rep. Prog. Phys., 68, 2859, (2005).

[15] C.-O. Ng \& C.Y. Wang, Effective slip for Stokes flow over a surface patterned with two- or threedimensional protrusions, Fluid Dyn. Res., 43, 065504, (2011).

[16] J. Ou, B. Perot \& J.P. Rothstein, Laminar drag reduction in microchannels using ultrahydrophobic surfaces, Phys. Fluids, 16, 4635, (2004).

[17] J. Ou \& J.P. Rothstein, Drag reduction and $\mu$-PIV measurements of the flow past ultrahydrophobic surfaces, Phys. Fluids, 17, 103606, (2005).

[18] J.R. Philip, Flows satisfying mixed no-slip and no-shear conditions, J. Appl. Math. Phys. (ZAMP), 23, 353-372, (1972).

[19] D. Queré, Wetting and roughness, Ann. Rev. Fl. Mech., 38, 71-99, (2008).

[20] J. P. Rothstein, Slip on superhydrophobic surfaces, Ann. Rev. Fluid Mech., 42, 89-109, (2010).

[21] M. Sbragaglia \& A. Prosperetti, A note on the effective slip properties for microchannel flows with ultrahydrophobic surfaces, Phys Fluids, 19, 043603, (2007).

[22] A. Steinberger, C. Cottin-Bizonne, P. Kleimann \& E. Charlaix, High friction on a bubble mattress, Nature Mater., 6, 665, (2007).

[23] H.A. Stone, A.D. Stroock \& A. Ajdari, Engineering flows in small devices: Microfluidics toward a lab-on-a-chip, Ann Rev Fluid Mech, 36, 381 (2004).

[24] A.D. Stroock, S.K. Dertigner, G.M. Whitesides \& A. Adjari, Patterning flows using grooved surfaces, Anal. Chem., 74, 5306-5312, (2002).

[25] C. J. Teo \& B. C. Khoo, Flow past superhydrophobic surfaces containing longitudinal grooves: effects of interface curvature, Microfluid Nanofluid, 9, 499-511, (2010). 